\title{
THE EPITHELIAL INTEGRITY IS PRESERVED DURING PARTICLE EXCHANGE ACROSS THE EPITHELIUM BY MACROPHAGES AND DENDRITIC CELLS
}

\author{
F. Blank, M. Wehrli, O. Baum, P. Gehr and B. Rothen-Rutishauser \\ Cell and Molecular Biology Young Scientist Travel Award, sponsored by AstraZeneca \\ Institute of Anatomy, University of Bern, Bern, Switzerland
}

WINNING ABSTRACT: The epithelium of the human airway wall serves as structural and functional barrier against inhaled and deposited particulate antigen. Recently we have shown that human blood monocyte-derived dendritic cells (MDDC) and human blood monocytes derived macrophages (MDM) collaborate as sentinels against foreign particulate antigen by building a transepithelial interacting cellular network. Although the tight junction belt was penetrated by processes of MDDC and MDM to transfer particles across the epithelium, the monolayer integrity of the epithelial cells was not affected. These results brought up two main questions: (1) How is the epithelial integrity preserved? (2) If MDM and MDDC make a direct contact by their processes can they exchange particles?

The presence of tight junction and adherens junction mRNA and proteins in MDM and MDDC monocultures was determined by RT-PCR, and immunofluorescence. Living co-cultures of MDM and MDDC exposed to particles (1 mm in diameter) were investigated by laser scanning microscopy.

We found that MDDC as well as MDM express adherens junction and tight junction mRNA and proteins as shown by RT-PCR and SDS-Page. Furthermore, performing live cell experiments we have seen that MDDC and MDM moved towards each other and that particles were transferred from MDM to MDDC. Interestingly, MDDC also made contacts with other MDDC and we observed particle exchange between them as well.

We may conclude from these findings that MDM and MDDC can form tight junction and adherens junction-like structures with epithelial cells to preserve the epithelial integrity during particle translocation across the epithelium. In addition, we demonstrate an exchange of particles from MDM to MDDC and MDDC to MDDC which supports our hypothesis that MDM and MDDC collaborate as sentinels.

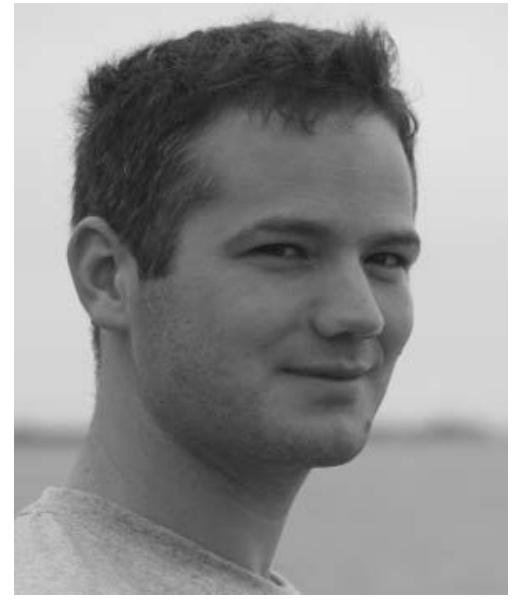

Fabian Blank

Institute of Anatomy, University of Bern, Bern, Switzerland

\section{MY JOB AND THE UNIT IN WHICH I WORK}

After completing my PhD, entitled "Cellular interplay in an optimized cell culture model of the airway wall upon exposure to fine particles", at the Division of Histology of the Institute of Anatomy at the University of Bern (Bern, Switzerland), I now

STATEMENT OF INTEREST: None declared. work as a postdoctoral researcher within the same group. Our main interest is the interaction of particles with cells in the lung and how cellular interplay is effected upon particle exposure. We are particularly interested in possible mechanisms which help antigen particles in the fine- $(0.1-2.5 \mu \mathrm{m})$, ultrafine- or nano-range $(<0.1 \mu \mathrm{m})$ to evade the molecular and cellular barrier components of the human lung. In recent years, our work has focused on the direct toxic effects of nanoparticles (nanotoxicology) on cells of the human lung. Our group maintains close collaborations with research groups on national and international levels, providing us with access to more sophisticated particle exposure techniques to study the effects of particles on cultured cells.

My research is carried out under the guidance of Prof. Peter Gehr and in close collaboration with Dr Barbara RothenRutishauser. My work particularly focuses on the interplay of cells of the defence system (i.e. monocyte-derived macrophages (MDM) and monocyte-derived dendritic cells (MDDC)) upon exposure to different sized fluorescent polystyrene particles. Cell-particle and cell-cell interactions, as well as subcellular localisation of particles, are monitored using conventional confocal laser scanning microscopy or live confocal laser scanning microscopy (using an incubation chamber for the cells combined with the confocal laser scanning microscope).

Besides my research activities, I am involved in supervising confocal laser scanning microscopy users and in maintaining the confocal microscope. Furthermore, all academic members of our group are involved in teaching medical students, giving 
lectures, courses and tutorials in anatomy histology, and supervising tutorial groups.

\section{MY WINNING ABSTRACT AS PART OF MY RESEARCH}

A series of structural and functional barrier components in the human airway wall prevent inhaled particulate matter (PM) from translocation into the tissue below the lung epithelium and, consequently, from entering the human body. These components include: 1) the surfactant film; 2) the liquid lining layer including the mucociliary escalator; 3 ) highly phagocytic macrophages at the luminal side of the lung epithelium; 4) the epithelium sealed with tight junctions; and 5) dendritic cells as specialised antigen presenting cells within and the base of the epithelium and the basal lamina (fig. 1) [1]. However, despite these barriers there are still particles that are translocated through the lung epithelium and a number of studies show that respiratory diseases caused by ambient PM are frequent and increasing in prevalence. Therefore, it is essential to investigate how and when inhaled PM evades these mechanisms of protection.

During my PhD thesis I worked with a triple-cell co-culture model, which represents the three most important cellular barrier components of the human airway wall: 1) the airway epithelium; 2) the airway macrophages located on top of the epithelium; and 3) the intra- or subepithelial dendritic cells [1, 2]. This in vitro model consists of a monolayer of a human epithelial cell line, either A549 (alveolar type II-like) cells or 16HBE14o (bronchial) cells, grown on a microporous membrane in a two-chamber system with MDM placed on top and MDDC placed underneath the epithelial monolayer (fig. 2) [1]. In the first step, I optimised the triple-cell, co-cultures by culturing the cells at the air-liquid interface in order to obtain a more realistic exposure set-up in which particles could be directly sprayed on to the co-cultures cell surface. For this reason the culture medium was removed from the upper chamber and the co-cultures were exposed to air for $24 \mathrm{~h}$ before particle exposure. The cell viability and the epithelial monolayer integrity were tested. The air-exposed epithelial cells maintained the monolayer structure for 2 days, expressed tight junctions and developed a transepithelial electrical resistance of $\sim 500$ and $\sim 200 \Omega \cdot \mathrm{cm}^{2}$ for $16 \mathrm{HBE} 140$ and A549 co-cultures, respectively. In a further step, fluorescently labelled $1 \mu \mathrm{m}$ polystyrene particles were sprayed on to the apical side of the air-exposed co-cultures using a microsprayer.

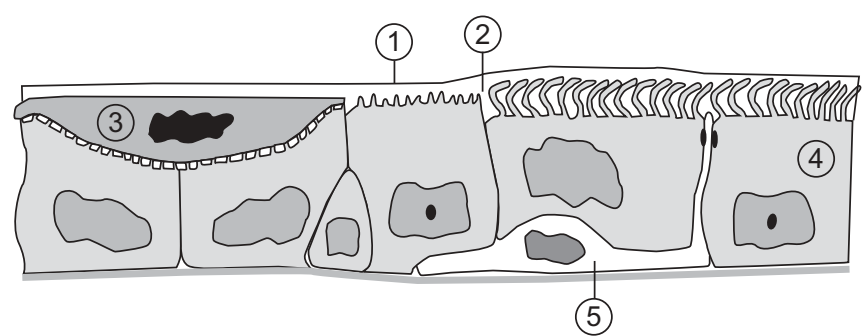

FIGURE 1. The barrier components of the human airway wall. 1) Surfactant film; 2) liquid lining layer covering the lung cells; 3) macrophages (specialised phagocytes); 4) epithelium sealed with tight junctions; and 5) dendritic cells (specialised antigen presenting cells), inside and underneath the epithelium. Adapted from [1] with permission from the publisher.
Particle location within the cultures and the cellular interplay was studied $10 \mathrm{~min}, 4 \mathrm{~h}$ and $24 \mathrm{~h}$ after particle exposition. For this investigation cultures were fixed and processed either for analysis with confocal laser scanning microscope or for analysis with the transmission electron microscope. In cocultures exposed to $1-\mu \mathrm{m}$ polystyrene particles, we found MDDC extending processes between epithelial cells through the tight junction to collect particles in the "luminal space" and transporting them inside the cytoplasmic processes between epithelial cells across the epithelium, or MDDC transmigrating through the epithelium to the apical side to take up particles on the epithelial surface. Furthermore, MDDC interacted with particle-loaded MDM on top of the epithelium and with other MDDC within or beneath the epithelium, forming a cellular network to take over particles (fig. 3) [1].

In the co-cultures, significantly more MDDC were involved in particle uptake when the epithelial barrier was made of A549 cells compared to MDDC with 16HBE14o cells as a barrier 10 min (mean \pm SD $23.9 \pm 14.3$ versus $9.5 \pm 10.4 \% ; \mathrm{p} \leqslant 0.05$ ) and $4 \mathrm{~h}(42.1 \pm 16.2$ versus $14.6 \pm 11.4 \% ; \mathrm{p} \leqslant 0.001)$ after particle exposition. In contrast, the MDM in co-culture with A549 cells showed a significantly lower involvement in particle uptake compared to co-cultures with $16 \mathrm{HBE} 14$ o cells $10 \mathrm{~min}(12.8 \pm 8.1$ versus $42.8 \pm 23.0 \% ; \mathrm{p} \leqslant 0.05)$ and $4 \mathrm{~h} \quad(57.4 \pm 27.7$ versus $82.7 \pm 19.1 \%$; $\leqslant 0.05)$ after particle exposition. Thus, in coculture with A549 cells, the higher involvement of MDDC in particle uptake reduced the particle burden to be taken up by MDM. It is probable that the low transepithelial electrical resistance of $\sim 200 \Omega \cdot \mathrm{cm}^{2}$ of the A549 epithelium facilitated the intervention of MDDC in particle uptake.

Thus, MDM and MDDC appeared to act as collaborating sentinels against inhaled particulate material by forming a dense transepithelial network of interconnected cytoplasmic processes. Measurement of transepithelial electrical resistance before and after particle exposure showed no significant lowering of transepithelial electrical resistance after particle exposure, i.e. tight junctions and, therefore, the epithelial integrity remained unaffected. Studies are currently being carried out to investigate how cytoplasmic processes extended by MDM and MDDC can penetrate the epithelial tight junction, sealing the epithelial monolayer at the apical side

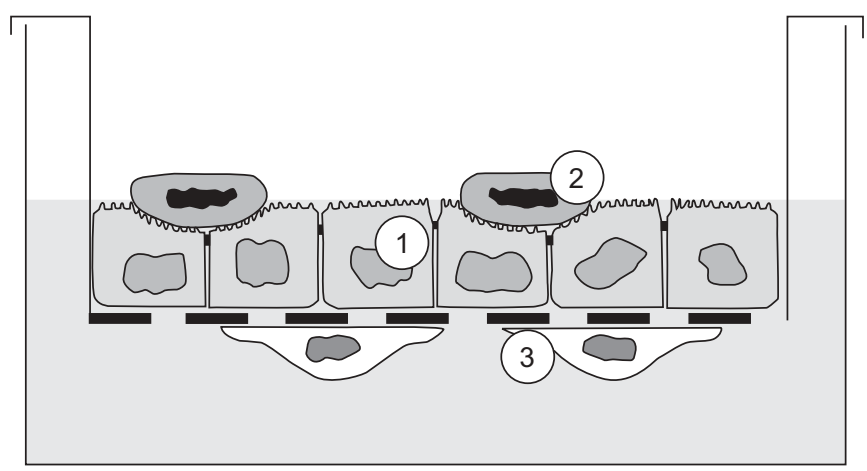

FIGURE 2. Triple-cell co-culture model of the human airway wall. 1) An epithelial monolayer is grown on a microporous insert membrane (- - - -). 2) Macrophages are placed on top of the epithelium. 3) Dendritic cells are placed underneath. Adapted from [1] with permission from the publisher 


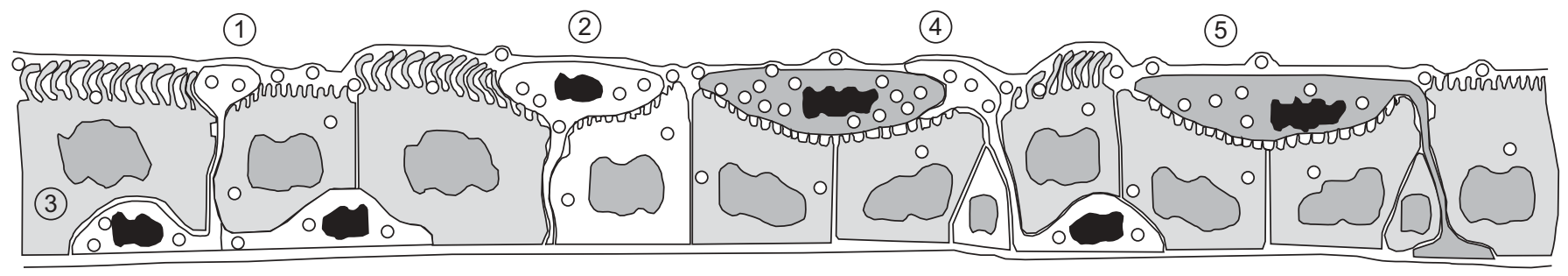

FIGURE 3. Cellular interplay at the particle-exposed airway wall. The following interactions of particle-exposed triple-cell co-cultures were observed: 1) extension of cytoplasmic processes by monocyte-derived dendritic cells (MDDC); 2) transmigration of complete MDDC; 3) particle transfer from MDDC to MDDC in or at the base of epithelium; 4) particle transfer from monocytes derived macrophages (MDM) to MDDC on top of epithelium; 5) MDM extending processes to the basal side of the epithelium Adapted from [1] with permission from the publisher.

without affecting the epithelial integrity. Furthermore, considerable effort is undertaken to study the effects of nanoparticles on the cellular interplay within our airway wall model and to compare the data with the findings revealed with fine particles (see above).

\section{MY RESEARCH AS PART OF MY WORKING GROUP/ RESEARCH TEAM}

With the advent of nanotechnology, the prospects of manufactured nanomaterials in many applications have progressed rapidly. The potential health effects of these nanoparticles, which have a diameter $\leqslant 0.1 \mu \mathrm{m}$, associated with human exposure are unknown. However, during the last few years scientists have found that exposure to combustion-derived ultrafine particles $(\leqslant 0.1 \mu \mathrm{m})$ is associated with pulmonary and cardiovascular diseases. The mechanisms by which ambient PM induce health effects are believed to include inflammation and oxidative stress. Due to the lack of knowledge regarding the health effects of nanoparticle exposure our group pays particular attention to these particles in order to compare their effects with the effects of ultrafine particles generated by anthropogenic activities. The comparison of manufactured nanoparticles to combustion-derived ultrafine particles suggests that the human health effects are similar.

We have three main ongoing projects which are linked. First, we want to know how the cells of the defence system proceed against inhaled nanoparticles (e.g. through collaboration or competition) in order to prevent them from entering the human body. Secondly, in case the nanoparticles are able to enter the tissue despite the cellular defence mechanisms, we are interested in their subcellular distribution addressing the following questions. In which cellular organelles can we detect particles? Is there an accumulation of nanoparticles in certain compartments, i.e. organelles of the cell? Is the subcellular localisation of a certain particle type dependent on material and/or size? Finally, based on the fact of their localisation in the cell, our research focuses on the potential cytotoxic or genotoxic effects of different nanoparticles with respect to material and dosimetry. Therefore, nanoparticles, especially those found in the nuclei of cells, are expected to induce DNA damage, while those found in the mitochondria are estimated to induce oxidative stress.

Most of our studies are conducted using the triple co-culture model. Once prepared, the triple cell co-cultures are exposed to different kinds of particles (fine, ultrafine) and different materials. The cellular and subcellular distribution of particles inside the co-cultures upon exposure is investigated using a wide range of advanced microscopic methods that are available at our institute, such as: transmission electron microscopy; electron energy loss spectroscopy; electron tomography for the subcellular localisation of nanoparticles and their elemental analysis; confocal laser scanning microscopy combined with deconvolution; and digital image reconstruction to quantify the amount of intracellular nanoparticles with respect to cell type and to the spatial localisation of nanoparticles within the tissue. Besides microscopic studies, the cytotoxic and genotoxic effects of particles on the cultured cells are investigated by monitoring the release of specific proinflammatory markers, using ELISA, and release of reactive oxygen species. Furthermore, the expression patterns of specific genes are studied using quantitative real-time PCR.

\section{REFERENCES}

1 Blank F, Rothen-Rutishauser B, Gehr P. Dendritic cells and macrophages form a transepithelial network against foreign particulate antigens. Am J Respir Cell Mol Biol 2007; 36: 669-677.

2 Rothen-Rutishauser BM, Kiama SG, Gehr P. Three-dimensional cellular model of the human respiratory tract to study the interaction with particles. Am J Respir Cell Mol Biol 2005; 32: 281-289. 